\title{
Efectividad de las técnicas de consejo breve
}

\author{
Rodríguez-Martos, A.
}

Pla d'Acció sobre Drogues. Institut Municipal de Salut Pública.

Enviar correspondencia: Alicia Rodríguez-Martos Dauer. PI. Lesseps, 1. 08023 Barcelona.

\section{RESUMEN}

En los últimos 20 años se ha investigado extensamente la eficacia de las intervenciones breves, denominación que designa una serie de intervenciones marginales con respecto a los programas terapéuticos tradicionales y que se caracterizan por su brevedad y concisión. Más de 40 estudios y diversos metaanálisis avalan la eficacia de estas intervenciones, cuya rentabilidad superaría a cualquier otra intervención en el medio inespecífico. Aunque su efectividad (resultados en condiciones naturales) y generalización no son tan evidentes como su eficacia en condiciones experimentales, su potencial repercusión sobre la Salud Pública hace que profesionales y autoridades sanitarias se esfuercen en su difusión y promoción. Pero, ¿nos hallamos ante una panacea? La diversidad de intervenciones agrupadas bajo el común denominador de intervenciones breves y la disparidad de estudios, a menudo difícilmente comparables, obligan a una revisión crítica que cuestione también su validez. Se concluye que la intervención breve es en conjunto eficaz, efectiva y eficiente, sobre todo en Atención Primaria de Salud, aunque no todas las intervenciones son igualmente eficaces.

La ampliación del espectro terapéutico, aplicando intervenciones más sencillas a los bebedores de riesgo, no debería hacerse en detrimento de recursos específicos más complejos para los alcohol-dependientes. Por lo que respecta a la intervención breve propiamente dicha, debe seguirse investigando su aplicación óptima: qué ingredientes, duración y extensión, en qué medio, a cargo de qué profesional, para qué paciente.

Palabras clave: intervención breve, eficacia, efectividad, eficiencia, validez.

\section{SUMMARY}

Brief intervention (a series of non-traditional therapeutic interventions which are characterised by their shortness and conciseness) has been widely investigated in the last 20 years. More than 40 studies and several meta-analyses support the efficacy of these interventions, the cost-effectiveness of which is higher than any other non-specific intervention. Although their effectiveness (results in natural conditions) and generalisation are not as evident as their efficacy in research designs, their potential repercussion on Public Health justifies their spreading and promotion by professionals and health authorities. But, is it a panacea we are talking about? The variety of interventions gathered under the name "brief intervention" and the disparity of studies, often hardly comparable, compel us to make a critical review, including their validity. The conclusion is that brief intervention is altogether effective, efficacious and efficient, especially in Primary Health Care, but not every brief intervention is equally effective. Broadening the therapeutic spectrum by offering easy intervention to heavy drinkers shouldn't be done at the expense of reducing specific resources for alcohol-dependent patients. More research is still needed concerning the optimum use of brief intervention: which elements, duration, extension, setting, by which professional and for which patient.

Key words: brief intervention, efficacy, effectiveness, efficiency, validity. 


\section{INTRODUCCIÓN}

E n los últimos 20 años se ha avanzado mucho en el conocimiento de la eficacia relativa de distintos abordajes terapéuticos y se ha tomado conciencia de la necesidad de comprobar asimismo el coste-efectividad de las intervenciones. Miller y sus colegas ${ }^{1}$ evaluaron 33 modalidades terapéuticas, evidenciando la eficacia de las intervenciones breves, reiteradamente avalada por diversos metaanálisis ${ }^{2-4}$, aunque a veces aparezcan datos discordantes que impiden estimar de modo fiable su efecto medio ${ }^{5}$.

A lo largo de la presente exposición intentaremos responder a las siguientes cuestiones: ¿A qué nos referimos exactamente cuando hablamos de intervención breve? ¿Se ha comprobado su eficacia, efectividad y eficiencia? Es decir: ¿funciona tanto en condiciones óptimas, como en condiciones normales y presenta un adecuado coste-beneficio? ¿Son los estudios metodológicamente correctos, comparables y generalizables? En aras a rentabilizar un recurso barato e inespecífico, ¿no corremos el riesgo de confundir la intervención breve con una panacea?

\section{1. ¿QUÉ ES LA INTERVENCIÓN BREVE?}

Concepto. La intervención breve (IB)es ${ }^{6}$ una intervención limitada en el tiempo de duración siempre inferior a la de un tratamiento específico, que incluye una evaluación global, un asesoramiento breve, y un cierto seguimiento. Bajo esta denominación se incluyen una serie de intervenciones marginales con respecto a los programas y procedimientos terapéuticos tradicionales, que tienen como característica su brevedad y concisión y suelen ser realizadas por médicos, enfermeras, psicólogos o trabajadores sociales.

Objetivo: Promover, mediante una estrategia sencilla y barata, un cambio en la conducta problema identificada en un paciente que no busca tratamiento, activando sus propios recursos. En pacientes dependientes, la IB debería orientarse a preparar la derivación a tratamiento específico, dada la insuficiente evidencia de su eficacia en estos casos.

La IB cumple funciones de prevención secundaria y es inseparable del diagnóstico precoz.

Con el fin de evidenciar su pluralidad, analizaremos sus distintos tipos para revisar luego sus componentes.

\subsection{Tipos de IB}

Aunque genéricamente podemos englobar dentro de las IB todas las que se realizan de forma abreviada, en la práctica podemos agruparlas según dos características básicas: su contexto y su estructura.

Contexto. Heather ${ }^{7}$ considera que existen dos categorías principales de intervenciones breves según su contexto: 1) Intervenciones basadas en un servicio específico, propuestas ${ }^{6}$ como "esquema terapéutico básico" o alternativa a programas más amplios, y 2) Intervenciones de base comunitaria, las más comunes, que pueden ser realizadas de forma "oportunista", cuando se identifica al paciente que acude por otro motivo distinto del alcohol ${ }^{8-11}$, o reclutando a la población a través de anuncios, cartas o cuestionarios rutinariamente administrados desde un centro sanitario en el marco de un programa de promoción de salud ${ }^{12-14}$.

La intervención breve de base comunitaria tiene un papel proactivo y constituye una vía alternativa al tratamiento convencional para aquellas personas que no necesitan programa de deshabituación (bebedores de riesgo, con consumo perjudicial o dependencia incipiente) o que se niegan a acudir a un recurso específico. Las personas que más se beneficiarían de la IB serían las que se hallan en la fase de contemplación y, por tanto, ambivalentes ${ }^{15}$.

Estructura. Por su estructura podemos distinguir entre: 1) Intervención muy breve o 
mínima (IM): consiste en una única sesión de consejo simple $\left(5^{\prime}-10^{\prime}\right)$, con o sin soporte de material de autoayuda, y 2) Intervención Breve (IB): Consiste en una entrevista única (15-30 ') o extendida (2-3 visitas de refuerzo), material de autoayuda y evaluación al año. Promueve la reflexión (ambivalencia frente al cambio) y sugiere estrategias.

La evaluación, que precede a la IM O IB, suele hacerse en la misma sesión que el consejo, cuando se trata de intervenciones oportunistas.
Fundamento teórico. La IB parte de los modelos transteorético del cambio y motivacional de intervención, cuya integración se promueve ${ }^{16} \mathrm{y}$ explicaría la eficacia de las IB ${ }^{17}$.

Elementos activos. 32 estudios ${ }^{2}$ identifican 6 elementos comunes (acrónimo FRAMES $\left.{ }^{18}\right)$ considerados "ingredientes activos" de la intervención, aunque se desconozca su importancia relativa. (Tabla 1). El único elemento común a todas las intervenciones estudiadas $^{2}$ es el consejo o "esencia de las IB". La información despertaría la conciencia y facilitaría la motivación para el cambio.

\section{Tabla 1. Elementos activos de la Intervención Breve}

\section{Elementos activos (FRAMES) ${ }^{18}$}

Feedback (evaluación devuelta)

Responsability (responsabilidad)

Advice (consejo)

Menu (menú)

Empathy (empatía)

Self-efficacy (autoeficacia)

Fte.: Bien et al. $1993^{2}$

\section{Frecuencia con que aparece cada elemento en los estudios analizados*}

\section{RENDIMIENTO DE LAS IB}

\section{1. Eficacia de las IB}

La eficacia de las IB es consistente y viene avalada por múltiples estudios y metaanálisis. A pesar de la diversidad de diseños y resultados, existe una opinión consensuada por lo que respecta a su eficacia ${ }^{1-4}$ y rentabilidad para la mayoría de sujetos de riesgo. La IB logra reducciones significativas del consumo de alcohol (en torno al 25\% $\%^{8,-10,19}$ e incluso superior ${ }^{11,20}$, con una diferencia media del $15 \%$ con respecto a controles $8,11,19,22$, que puede llegar hasta el $24 \%$ en IB atípicas por su extensión ${ }^{20}$ ). El porcentaje de bebedores excesivos se reduce alrededor del $45 \%^{21}$ frente al $25-30 \%$ de los controles ${ }^{8,19,22}$ aunque puede haber reducciones superiores al $60 \%{ }^{8,11,23}$. Los episodios de 'atracones de con- sumo' (binge drinking) disminuyen en torno al $50 \%\left(45,67 \%{ }^{11}-74 \%{ }^{23}\right)$. La intervención breve logra disminuir la incidencia de problemas relacionados con el alcohol: problemas psicosociales (hasta un $85 \%)^{20}$; bajas por enfermedad (80\% en 4 años), días de hospitalización médica (en torno al $60 \%$ en 5 años) y mortalidad (50\% en 6 años) ${ }^{12}$; lesividad (47\%) y días de hospitalización por traumas (48\%), a los 3 años ${ }^{24}$. Los valores de GGT descienden de forma significativamente mayor en los pacientes a quienes se ha realizado una intervención breve que en los del grupo control. ${ }^{12,14,20,25-26}$. La IB no suele ser tan eficaz en mujeres, ${ }^{19.22,27,8}$ lo que pudiera obedecer a una disminución de consumo menor por partir de ingestas menos elevadas, a su mayor respuesta a la simple evaluación o simplemente a un tamaño insuficiente de la muestra. En cualquier caso, también hay estudios donde la mujer alcanza reducciones de significación 
equiparables al varón ${ }^{11}$. En los jóvenes varones la intervención desde el ámbito sanitario tiene menos posibilidades de éxito. ${ }^{22}$

La superioridad de las IB sobre las IM se ha cuestionado. Sería escasa y no estadísticamente significativa, según algunos estudios ${ }^{13-}$ 14,19,22, pero existen evidencias de un incremento en el rendimiento de la intervención paralelo a la intensidad, extensión o número de visitas de seguimiento $3,5,8,20,25,27-29$. Las intervenciones únicas (de cualquier duración, pero sin visitas de refuerzo) no presentarían ${ }^{5}$ una eficacia uniforme. El estudio de la OMS ${ }^{19,22}$ reveló que el rendimiento óptimo de la IM se da en quienes han sufrido un evento negativo reciente relacionado con el alcohol. La intervención breve parece especialmente recomendable en personas con consumo peligroso o perjudicial de alcohol. En personas con dependencia, la IB no siempre aporta mejorías estadísticamente significativas ${ }^{13,30}$, aunque puede facilitar la derivación ${ }^{15}$ y el cumplimiento terapéutico ${ }^{2}$. Cabe considerar una adscripción diferenciada de los pacientes, según sus características en cuanto a gravedad, experiencia de un evento negativo reciente ${ }^{22}$ y disposición para el cambio ${ }^{31}$ : 1) Intervención mínima: basta en los que tengan sólo un consumo de riesgo, hayan tenido una experiencia aversiva o estén preparados para el cambio; y 2) Intervención breve: bebedores de alto riesgo, quienes presenten problemas crónicos sin eventos aversivos recientes y quienes no se sientan preparados para el cambio.

\subsubsection{Eficacia de la IB en ámbitos diversos}

\section{Intervenciones breves en Atención Pri- maria de Salud (APS)}

Según los estudios que reúnen mayor grado de validez, ${ }^{8-10,19,22}$ existe una significativa reducción del consumo semanal en los varones frente a controles, pero no tanto en mujeres, aunque el tamaño de la muestra es a veces excesivamente reducido. ${ }^{9}$

\section{MRC Medical Research Council Study ${ }^{8}$}

En 47 centros de salud, 917 pacientes de ambos sexos que bebían más de $35 \mathrm{U} /$ semana (hombres) o más de $21 \mathrm{U} / \mathrm{semana}$ (mujeres) fueron reclutados mediante un cuestionario de salud y asignados aleatoriamente a un grupo control (sólo evaluación) o al grupo de tratamiento (15 minutos de consejo médico de reducción de consumo y hasta 4 visitas de refuerzo). Al año, los varones tratados habían reducido una media de 18,2 U/semana, frente a $8,1 \mathrm{U} / \mathrm{s}$, en los controles $(p<0,001)$. El porcentaje de bebedores excesivos se había reducido en un $43,7 \%$ en el grupo de intervención, frente al $25,5 \%$ en el grupo control. En la mujer, el grupo de tratamiento redujo su ingesta en 11,5 U/s, comparado con $6,3 \mathrm{U} / \mathrm{s}$ en el grupo control $(p<0,05)$ y el porcentaje de bebedoras excesivas disminuyó en un $47,7 \%$ frente al $29,2 \%$, en controles. La reducción del consumo, así como del porcentaje de bebedores excesivos fue inversamente proporcional al número de sesiones de consejo médico.

\section{Estudio de Colaboración de la OMS sobre Detección y Tratamiento de Proble- mas relacionados con el alcohol desde Atención Primaria de Salud ${ }^{19,22}$}

Ensayo clínico multicéntrico y transnacional que incluye 1.655 pacientes, reclutados en función de su consumo excesivo (semanal o episódico) en entornos muy diversos. Los pacientes fueron aleatoriamente asignados a grupo control (sólo evaluación), grupo de consejo simple (evaluación $+5^{\prime}$ consejo) y grupo de intervención breve (ídem+15' consejo y manual de autoayuda). A los 6 meses, el porcentaje de bebedores excesivos que había reducido su consumo medio fue del $40,8 \%$ y $40,3 \%$, respectivamente para los grupos de IM e IB, frente al $29 \%$ en los controles. EI grupo control de hombres control había reducido en un $10 \%$ el consumo medio, mientras que los grupos de IM e IB habían reducido un $37,8 \%$ y $31,9 \%$, respectivamente. Las mujeres redujeron su consumo en ambos grupos de forma no significativa. Aunque ni la adición de 15 minutos de asesoramiento y un manual de autoayuda, ni la aplicación de un programa 
de asesoramiento extendido (3 visitas en 6 meses) aportaron beneficios adicionales, la intervención breve fue superior al simple consejo en pacientes que no habían experimentado un problema reciente relacionado con el alcohol.

2).

\section{La experiencia española ${ }^{28-29,32-34}$ (Ver tabla}

En España, la IB en sus distintas modalidades ha ido incorporándose en las consultas de Atención Primaria, impulsada por la Sociedad Española de Medicina Familiar y Comunitaria y, más recientemente, por la introducción en Cataluña del programa Bebed Menos del estudio de Colaboración de la $\mathrm{OMS}^{32}$.

Fernández et al. ${ }^{33}$ realizan una IB sobre 152 varones con consumo $>21 \mathrm{u} / \mathrm{s}$ en 12 consultas de APS de Madrid. El estudio es un ensayo clínico controlado con asignación aleatoria y evaluación ciega. Se excluyen alcoholdependientes y no se interviene sobre el grupo control. El seguimiento se extiende 618 meses y se pierde en torno al $40 \%$ de la muestra. Los autores encuentran una reducción significativa del consumo en pacientes con un consumo de 35 o más unidades semanales, a los que se había aplicado un consejo breve (10') por parte de su MC apoyado con material didáctico. La recogida inicial de datos y el seguimiento -a los 9-10 meses- lo realizan enfermeras. La disminución conseguida en el grupo de intervención es significativa. El porcentaje de bebedores de riesgo pasa del $73,7 \%$ al $39,5 \%$ en el grupo de intervención. En el grupo control la disminución no es significativa.

El estudio EMPA ${ }^{28}$ incluye 139 varones detectados como bebedores de $>35 \mathrm{u} / \mathrm{s}$ en 16 consultas de APS de Aragón, Lérida y Navarra y excluye a los alcohol-dependientes. Se trata de un estudio clínico controlado con asignación aleatoria, pero sin evaluación ciega. No existe auténtico grupo control, siendo los presuntos controles objeto de consejo simple $\left(5^{\prime}\right)$ y visita de control al año. Al grupo experimental se le da material didáctico y se le programan vistas de seguimiento $(1,4,7,12$ meses). Se pierde una tercera parte de la muestra. Disminuyen significativamente tanto el consumo de unidades /semana ( $58,9 \%$ frente a $36,1 \%$ ) como el porcentaje de bebedores de riesgo ( $82 \%$ versus $47 \%$ )

El estudio EIBAL ${ }^{29}$ perfecciona y amplía el anterior. Se trata de un estudio multicéntrico que incluye 33 centros y 546 pacientes varones. Excluye dependientes. El estudio tiene diseño naturalístico y las intervenciones se distribuyen aleatoriamente por centros (controles o intervención breve), sin evaluación ciega. Tampoco aquí hay auténtico grupo control, recibiendo el así denominado grupo una IM (consejo simple de $5^{\prime}$ ), mientras que el grupo experimental recibe una IB (15') con material de apoyo y la oferta de visitas de refuerzo. A los 12 meses de seguimiento, se ha perdido casi el $50 \%$. La disminución del consumo (52\% frente a $32 \%$ ) y del porcentaje de bebedores excesivos $(67 \%$ frente a $44 \%$ ) son significativos frente al grupo control.

Cataluña participa en el Proyecto de Colaboración de la OMS ${ }^{19,22}$ cuyo programa "Bebed Menos" pretende diseminar la intervención breve en atención primaria de salud. Como paso previo a su implementación, actualmente en curso, se ha formado a los profesionales de la red de drogodependencias para que formen a sus colegas de atención primaria (modelo "formación de formadores"). Tras participar en el seminario, la actitud de los profesionales es muy positiva, tanto frente a las intervenciones breves como en relación con las tareas formativas y de asesoramiento, para las que dicen sentirse preparados $(67 \%)^{32}$.

Otra experiencia interesante y autóctona es la del Programa Cantabria de Intervención Breve en bebedores de riesgo no dependientes, que trabaja a partir de grupos psicoeducativos $^{34}$.

\section{Intervenciones breves de ámbito comu- nitario}

Sujetos reclutados mediante anuncio utilizando el Drinker's Ckeck-Up ${ }^{35}$ obtuvieron una reducción del consumo significativamente superior a los sujetos en lista de espera, 


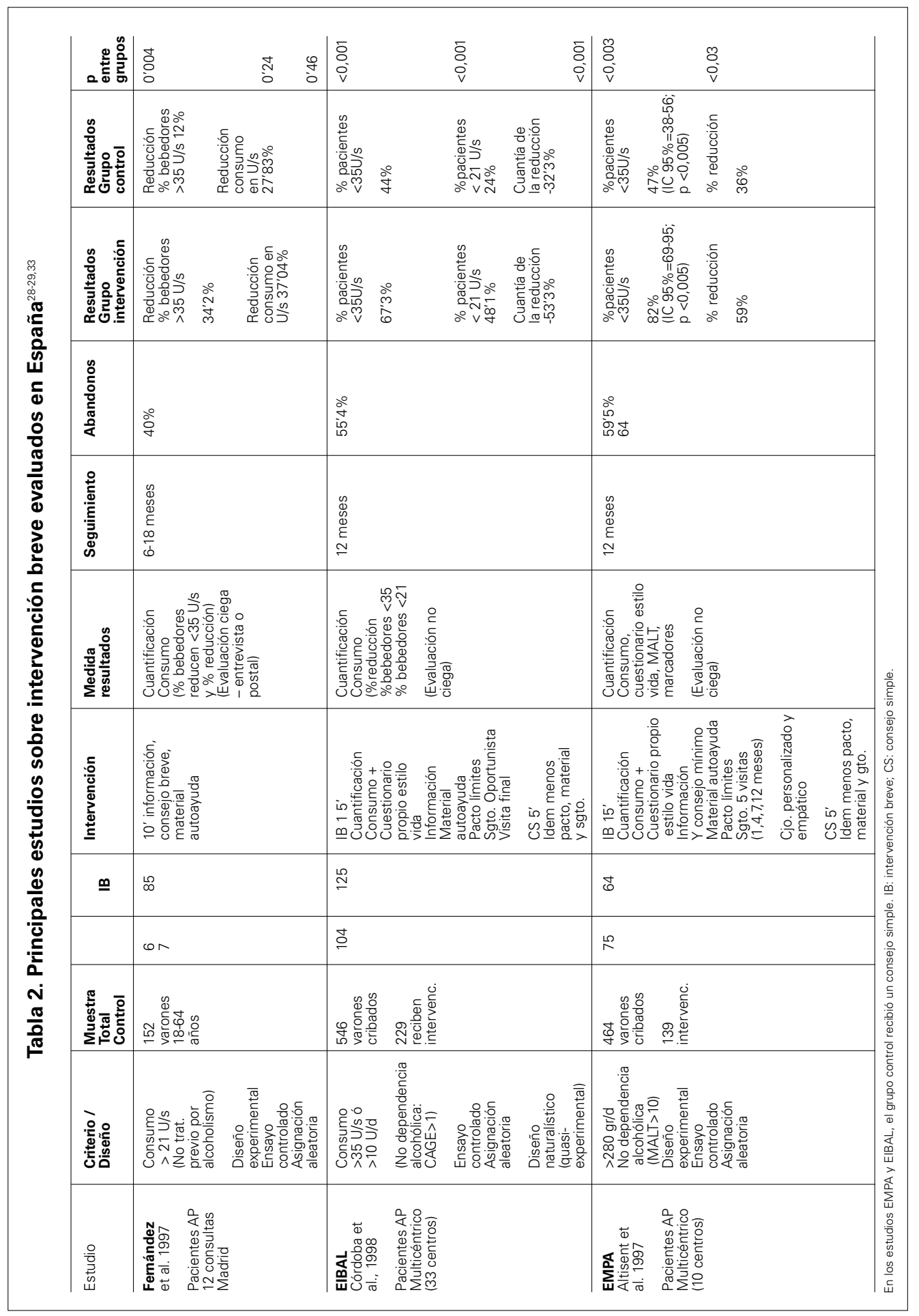


mantenida a los 18 meses, experiencia replicada años después. Heather et al. ${ }^{30}$ comprobaron la utilidad de enviar por correo un manual de autoayuda, significativamente superior al folleto educativo inespecífico, a los 6 meses. Al año, no había diferencias significativas entre el programa estructurado DRAMS, el consejo simple o la no-intervención: todos reducen, pero todos habían recibido una exhaustiva evaluación y completaron el estudio menos de la mitad.

Sujetos reclutados a partir de un examen de salud en una muestra aleatoria de población adulta de Estocolmo ${ }^{13}$ fueron distribuidos al azar en grupo control (consejo médico)y de intervención (3 o más visitas de su médico e información de sus valores de GGT como refuerzo). Al año, había mejorado más aunque sin diferencias significativas - el grupo de intervención, tratándose en el fondo de dos tipos de intervención (mínima y breve) más que de IB y control.

El estudio de Tromso ${ }^{14}$ selecciona a 338 sujetos a partir de una encuesta de salud y los distribuye aleatoriamente la muestra en grupo control, IM y IB. Al cabo de 1 año, se habían reducido significativamente el consumo de alcohol y la GGT en ambos grupos de intervención, sin diferencia significativa entre ellos.

\section{Intervenciones breves en el campo de los servicios hospitalarios}

En el ámbito hospitalario se ha comprobado la eficacia de las IB (reducción de consumo y de problemas) en pacientes que habían acudido por motivos diferentes al alcohol, ${ }^{36-37}$ con mejor rendimiento de las estrategias motivacionales en pacientes "no preparados" para cambiar. ${ }^{36}$

Kristenson et $\mathrm{al}^{12}$ comprueban la superioridad de una IB con visitas reiteradas de refuerzo frente a grupo control (al que se informaba de su situación) en el marco de un programa de promoción de salud de Malmö (Suecia).

\section{Intervenciones breves en el campo de los servicios hospitalarios de urgencias}

Chafetz $^{38}$ fue el primero en concebir una intervención breve destinada a motivar para la derivación a los alcohólicos atendidos en urgencias médicas, encontrando diferencias muy significativas entre los sujetos que recibieron la IB y el grupo control. En el Boston Medical Center, aplican con éxito el Project ASSERT. 15,39 , en pacientes atendidos en urgencias, donde asistentes sociales formadas realizan la "entrevista breve de negociación" (detección, intervención y derivación). El reducido tamaño de la muestra y las elevadas pérdidas para el seguimiento (78\%) restan validez al resultado (favorable en el $50 \%$ de casos).

\section{Intervenciones breves en el campo de la traumatología}

La IB sobre pacientes lesionados en la fase aguda de su tratamiento se considera clave para motivarlos para un cambio que reduzca su riesgo futuro ${ }^{24,26,40-41}$.

Antti-Poika ${ }^{26}$ informa acerca de una intervención, por parte de una enfermera entrena$\mathrm{da}$, en 120 varones ingresados por lesiones en un departamento de ortopedia y traumatología. Se administraba un consejo breve de reducción o abstinencia, según los casos, y se entregaba un pequeño manual de autoayuda. En las visitas de seguimiento (una, dos y hasta tres veces) por parte de un departamento especializado, se repitió el consejo. A los 6 meses, el $45 \%$ de pacientes sobre los que se había intervenido habían reducido o suprimido su consumo de alcohol, frente al $20 \%$ de los controles. Paralelamente se había producido una reducción en los valores de la GGT.

El evento aversivo de un accidente atribuible al alcohol constituye un "momento receptivo" ${ }^{42}$ y el periodo agudo post-traumático representa la mejor "ventana de oportunidad" para intervenciones educativas. Sommers et al ${ }^{40-41}$ trabajan en un estudio sobre intervenciones breves en accidentados de tráfico, (distribución aleatoria en grupos control, de IM e IB) cribados en el servicio de urgencias de un hospital traumatológico. Al año, aparece una reducción en todos los 
parámetros de consumo y en todos los grupos, aunque significativamente superior en el grupo de IB, que se mantiene tras haber incrementado ligeramente el consumo entre los meses 1-3, en tanto que en los otros grupos, el consumo ha seguido aumentando a lo largo del año, sin alcanzar la ingesta basal.

Otro estudio realizado en un centro de traumatología, en el que se sigue trabajando, es el de Gentinello et al. ${ }^{24}$ con distribución aleatoria entre grupo control y de IB indica que, a los 12 meses el grupo experimental había reducido su consumo significativamente más que el control $(p=0,03)$, especialmente en pacientes con problemas poco graves de alcohol $(p<0,01)$. La reducción más aparente sobrevino a los 6 meses. A posteriori, el grupo control reanudó su consumo basal en tanto que los que recibieron la intervención continuaron disminuyendo el consumo. Asimismo se redujo en un $47 \%$ la lesividad subsidiaria de atención hospitalaria y en un $48 \%$ la hospitalización por traumas a los 3 años.

En España, un estudio en curso investiga la eficacia de las intervenciones breves desde un hospital traumatológico de Barcelona. ${ }^{43}$

\section{Intervenciones breves en clínicas especi- ficas para problemas de alcohol}

En este contexto, los estudios sobre IB suelen hacerse para compararlos con tratamientos más prolongados ${ }^{2}$. En general, no se observan diferencias entre intervenciones breves y extensas. Sin embargo, Chick et al. $1988^{25}$ comunican que quienes hacen tratamiento convencional funcionan mejor a los 2 años, aunque la abstinencia no sea superior a los que recibieron consejo (simple o ampliado). La decisión del paciente de hacer algo con su bebida sería más importante que el tipo de intervención ofrecida ${ }^{25}$ y la experiencia de los terapeutas también puede jugar un papel en ambos grupos que, además, suelen ser objeto de una extensa evaluación inicial ${ }^{44}$

\section{Intervenciones breves en el ámbito labo- ral}

Aunque las experiencias en este campo son incipientes ${ }^{45}$, el mundo laboral parece un entorno adecuado para actividades de promoción de salud, al permitir identificar e intervenir sobre individuos difíciles de alcanzar en otros ambientes, tratándose sobre todo de población joven que no va mucho al médico.

\subsection{Efectividad-repercusión sobre la salud pública}

Los ensayos sobre eficacia y sobre efectividad se diferencian en que los primeros se refieren al resultado de la intervención en condiciones óptimas y maximizan la validez interna del procedimiento, mientras que los segundos se destinan a evidenciar el resultado del tratamiento en el mundo real y, por tanto, destinados a aumentar la validez externa del mismo.

Para que una intervención repercuta sobre la salud pública ha de ser eficaz y efectiva.

Probablemente ${ }^{46}$ la eficacia de la IB como intervención rutinaria ha sido exagerada. Richmond y Anderson ${ }^{47}$ reconocían la imposibilidad de afirmar que la eficacia de la IB demostrada en estudios controlados y aplicada por profesionales voluntariamente enrolados y especialmente entrenados ${ }^{22}$, podía ser extrapolable al rendimiento global de la IB aplicada desde la AP de forma generalizada. Por otra parte, la elevada atrición (media de $70,6 \%$ ) que presentan los estudios realizados en AP invita a la prudencia a la hora de extraer conclusiones, pues los sujetos estudiados podrían ser los candidatos más susceptibles a la intervención ${ }^{48}$.

Los métodos de reclutamiento oportunista o naturalista (paciente que acude a consulta y es detectado como bebedor de riesgo) son más representativos y aplicables que aquellos que reclutan a los bebedores vía correo, anuncios etc. ${ }^{47}$, como evidencia el metaanálisis de Kahan et al. ${ }^{4}$ al analizar cada estudio según criterios de generalización previamente seleccionados.

Aunque existen más pruebas de su eficacia que de su efectividad, parece lógico atribuir a la IB un enorme impacto potencial sobre la salud pública ${ }^{2,422}$, si se aplicara de forma rutinaria. 
La dicotomía entre un modelo individual y comunitario de prevención tiende a difuminarse. Las IB, junto con el tratamiento de enfermos, debería tener una repercusión sobre la salud pública tanto más manifiesto cuanto más integrado esté el programa en los recursos comunitarios. Uno de los principales obstáculos para transferir la experiencia experimental a la práctica es la necesidad de asistencia técnica para los implicados, habiéndose sugerido la elaboración de guías didácticas adaptadas a diferentes entornos ${ }^{49}$. Desde el punto de vista de la Salud Pública, la diseminación de las IB debería formar parte de un paquete de 4 elementos ${ }^{49}$ : educación preventiva, cribado, intervención breve y derivación.

\subsection{Eficiencia}

La IB sería, a juicio de Holder ${ }^{50}$ la modalidad de intervención más rentable en el medio sanitario inespecífico, por ser la que obtiene mejor balance coste-beneficio para la mayor parte de sujetos de riesgo ${ }^{49}$. La rentabilidad económica de la IB dependerá de cómo se aplique y será tanto mayor cuanto más se focalice en una población diana seleccionada por su alto riesgo: por ej,: accidentados de tráfico atendidos en urgencias, ya que en dicho contexto se detectarán más bebedores de riesgo que en un abordaje poblacional ${ }^{49}$.

\section{3. ¿SON LOS ESTUDIOS METODOLÓGICA- MENTE CORRECTOS?}

Edwards había afirmado en una reunión en la Royal Society of Medicine, en Londres (1988) que, cuanto mejor era un estudio, menor era la probabilidad de que apareciera un efecto significativo del tratamiento. Entre los defectos más frecuentes, cabe señalar la falta de seguimiento ciego, la elevada atrición y la medida de resultados, así como la contaminación de los grupos control por exhaustivas evaluaciones. Los estudios de metaanálisis son el procedimiento más adecuado a la hora de analizar la eficacia de una determinada estrategia terapéutica, ya que tratan de analizar separadamente los estudios - según diferentes parámetros y ambiente en el que se han realizado $2,4,5$ - o aplican siquiera criterios comunes que permitan comparar los estudios seleccionados.

El metaanálisis de Bien et al. ${ }^{2}$ analiza separadamente: estudios en ámbito sanitario, estudios comunitarios con pacientes autoderivados y la IB en contextos terapéuticos.

Desde el punto de vista metodológico, se realiza una valoración individualizada de la calidad de cada ensayo basada en 12 criterios (Tabla 3) siempre a cargo de al menos dos evaluadores independientes. La escala, cuya pun-

\section{Tabla 3. Escala de Calidad Metodológica (Metaanálisis de Bien et al.)}

\section{Concepto}

Puntuación
1. Asignación (por ej., aleatorización)
$0-4$
2. Control de calidad tratamientos administrados
$0-1$
3. Grado de cumplimiento del seguimiento
$0-2$
4. Duración del seguimiento
$0-2$
5. Seguimiento personal/telef. v. Cuestionario
$0-1$
6. Inclusión de entrevistas colaterales
$0-1$
7. Inclusión verificación objetiva de autoinforme
$0-1$
8. Consignación de abandonos de tratamiento
$0-1$
9. Consignación de atrición en seguimiento
$0-1$
10. Entrevista ciega
$0-1$
11. Análisis estadístico adecuado
$0-1$
12. Replicación multicéntrica
$0-1$
Total:
0-17 puntos

Fte: Bien et al., 1993² 
tuación máxima es de 17 puntos, valora sobre todo el procedimiento de asignación (4 puntos para la asignación aleatoria) el cumplimiento del seguimiento, (2 puntos al seguimiento del $85-100 \%$ en cualquier momento a partir de los 3 meses), y la duración del seguimiento, otorgándose 2 puntos a los de 12 o más meses).

Metaanálisis de Kahan et al. ${ }^{4}$ : Kahan et al. realizan un metaanálisis incluyendo solamente estudios sobre IB aplicada por médicos y los evalúan siguiendo criterios de validez y generalización (ver Tabla 4) elaborados por los investigadores y aplicados, en cada caso, por al menos 2 investigadores distintos.

Metaanálisis de Ballesteros ${ }^{51}$ : Este metaanálisis revisa los estudios españoles sobre eficacia de la IB en bebedores excesivos atendidos en centros de salud por motivos distintos al alcoho| ${ }^{28-29,33}$, destacando la calidad de los estudios de Fernández et al, $1997^{33}$ y Altisent et al. ${ }^{28}$ (16 puntos según criterios de validez de Kahan et al. ${ }^{4}$ ). (Tabla 2)

Metaanálisis de Poikolainen ${ }^{5}$ : Los metaanálisis no suelen plantearse la distinción entre intervenciones breves únicas (entre 5 y 20 minutos de duración) e intervenciones breves extendidas (varias visitas). Éste es el primer metaanálisis que se plantea dicha distinción en población de Atención Primaria de Salud. Otra característica diferencial de este metaanálisis es que se centra en dos medidas de efecto principales, separadamente

Tabla 4. Criterios de calidad de Kahan et al.

\section{Criterios de validez}

Puntos

1. Asignación aleatoria

2. Control de factores pronósticos

3. Exclusión de pacientes con dependencia

4. Considerar pérdidas como recaída

5. Análisis de la intención de tratar

6. Seguimiento

7. Duración del ensayo

8. Información completa sobre consumo

9. Verificación de consumo autorreferido

10. Inclusión de datos sobre morbi-mortalidad

11. Evaluación ciega respecto a asignación

$0-2$

12. Contaminación grupo control

$0-2$

13. Intervención complementaria

$0-2$

14. Adecuación del tamaño de la muestra (varones o mixta)

$0-2$

15. Adecuación del tamaño de la muestra (mujeres)

TOTAL

\section{Criterios de generalización}

Puntos

1. Procedencia de la muestra

2. Duración entrenamiento

3. Duración de la intervención

4. Criterios de exclusión

5. Reclutamiento

6. Inclusión de mujeres

7. Agente

$$
\text { TOTAL }
$$


consideradas: el consumo medio de alcohol y el valor GGT. Partiendo de la búsqueda de estudios en las bases de datos más importantes y de revisiones previas, los investigadores encuentran 7 estudios ${ }^{8-11,14,27,30}$ sobre población de Atención Primaria. Algunos tenían un grupo experimental frente a otro control; otros, un control frente a dos intervenciones. Todos estudiaban la intervención en ambos sexos, aunque en 2 de ellos ${ }^{14,30}$ no se analizaba separadamente el resultado por sexos. De los 7 estudios, sólo los de Oxford, sólo hombres y Oxford, sólo mujeres ${ }^{10,9}$ y Fleming ${ }^{11}$ presentaban una asignación aleatoria. El seguimiento ciego se daba en todos, menos el Estudio Tromso ${ }^{14}$. La atrición más elevada se daba en los estudios de Oxford ${ }^{9-10}$ y en el Alcoscreen ${ }^{27}$. (Ver Tabla 5)

\section{Tabla 5. Diferencia entre la ingesta alcohólica de pacientes intervenidos versus controles (seguimiento) ${ }^{5}$}

\begin{tabular}{lllc}
\hline Estudio & Sexo & Tipo intervención & $\begin{array}{c}\text { Diferencia consumo } \\
\text { (gramos /semana) }\end{array}$ \\
\hline DRAMS & $\mathrm{H}(75 \%)$ & Breve (única) & -89 \\
DRAMS & $\mathrm{H}(75 \%)$ & Extensa & -109 \\
MRC & $\mathrm{H}$ & Extensa & $-93^{* *}$ \\
MRC & $\mathrm{M}$ & Extensa & $-54^{* *}$ \\
Oxford & $\mathrm{M}$ & Breve & -23 \\
Tromso & $\mathrm{H}(86 \%)$ & Breve & $-165^{* *}$ \\
Tromso & $\mathrm{H}(86 \%)$ & Extensa & $-180^{* *}$ \\
Oxford & $\mathrm{H}$ & Breve & -77 \\
Richmond & $\mathrm{H}$ & Breve & +31 \\
Richmond & $\mathrm{H}$ & Extensa & +54 \\
Richmond & $\mathrm{M}$ & Breve & +27 \\
Richmond & $\mathrm{M}$ & Extensa & +20 \\
TrEAT & $\mathrm{H}$ & Extensa & $-39^{* * *}$ \\
TrEAT & $\mathrm{M}$ & Extensa & $-62^{* * *}$ \\
\hline
\end{tabular}

${ }^{*} p<0,001$

Fte.: Poikolainen, $1999^{5}$

Los estudios MRC ${ }^{8}$ y de Oxford ${ }^{9-10}$ son los únicos que analizan los resultados en función de la Intención de Tratamiento. El estudio DRAMS ${ }^{30}$ evalúa la intervención muy breve frente a extensa; el MRC siempre realiza una intervención extensa, que compara con controles. Los estudios de Oxford ${ }^{9-10}$ evalúan intervenciones únicas y breves, mientras que el estudio australiano ${ }^{27}$ compara una intervención muy breve con otra extensa, tanto en hombres como en mujeres. Al agrupar los datos relativos a las intervenciones extensas, Poikolainen ${ }^{5}$ concluye que tienen una eficacia uniforme, sobre todo en la mujer, lo que no puede decirse de las intervenciones únicas, cuya eficacia no sería uniforme, impidiendo su significativa heterogeneidad estadística determinar su efecto medio.

En conjunto, a partir de los diversos metaanálisis publicados ${ }^{1,2,3,45,51}$, podemos destacar como de máxima calidad y obligada referencia los siguientes estudios: $M R C^{8}$, Oxford hombres ${ }^{10} \mathrm{OMS}^{19,22,}$ Oxford mujeres ${ }^{9}$, DRAMS, ${ }^{30}$ AlcoScreen ${ }^{27}$ y Estudio TrEAT ${ }^{11}$

\section{4. ¿SON LOS ESTUDIOS COMPARABLES?}

Es difícil comparar estudios por su heterogeneidad $^{52}$, sobre todo cuando se mezclan 
intervenciones breves a cargo de médicos de atención primaria con las realizadas por especialistas. Dicha heterogeneidad puede asimismo sesgar los metaanálisis ${ }^{5}$ siendo el sesgo de selección el más importante ${ }^{53}$. Lo que se publica no siempre es representativo de lo que se investiga y lo que se publica puede adolecer de sesgos de evaluación o redacción, destacando los resultados favorables. Por otra parte, dada la vía de localización de estudios, básicamente en las bases de datos MEDLINE y EMBASE, existe también un sesgo a favor de la literatura de habla inglesa. Otro sesgo puede ser el de la calidad de la investigación, sujeto a opinión personal, si no se posee un cuestionario para evaluarla. Y, si incluso los análisis mejor diseñados encuentran dificultades a la hora de comparar estudios de forma fiable, ¿cómo puede el simple lector sacar conclusiones unívocas cuando revisa la literatura? Heather ${ }^{54}$ advierte en contra de una interpretación simplista de los resultados. Ni todas las IB son iguales, ni los individuos a las que se aplican son siempre comparables (intervenciones oportunistas en bebedores que no buscan tratamiento versus aplicadas a quienes consultan por problemas de alcohol). La multiplicidad de factores en juego nunca permitirá poner a todas las intervenciones "en el mismo saco".

\section{CONCLUSIÓN}

A pesar de la multiplicidad y disparidad de factores que convierte a los estudios en heterogéneos y difícilmente comparables, existe evidencia suficiente de que la mejoría de los sujetos sometidos a IB no es sólo atribuible ${ }^{2}$ a artefactos del diseño o de las características de la población (evaluación inicial, motivación, respuesta a la auto-monitorización, mero paso del tiempo...). En conjunto, la eficacia, efectividad y eficiencia de la intervención pueden considerarse demostradasespecialmente en AP- aunque la importante atrición pueda sesgar los resultados ${ }^{48}$. No obstante, nos engañaríamos si creyéramos que todas las intervenciones breves son igualmente eficaces ${ }^{5}$. Su efecto no es uniforme y deberíamos encontrar la respuesta a preguntas relativas a su rendimiento óptimo: ¿Qué intervención (ingredientes, duración y extensión óptimos)? ¿Para qué pacientes? ¿Administrada por qué profesional? ¿En qué medio?

En conjunto, los estudios sobre la IB en el medio sanitario inespecífico se han hecho comparándola con la no intervención e indican la eficacia de la IB en cuanto a la reducción del consumo y/o de problemas. No ha podido dilucidarse de forma definitiva si el simple consejo es tan válido como una IB más extensa debido a la escasa comparabilidad de los estudios. En condiciones naturales los resultados no parecen tan espectaculares ni aplicables a todos los pacientes de AP. Los mejores resultados de la IB se dan en bebedores excesivos de mediana edad ${ }^{48}$ y podrían optimizarse adecuando la intervención al paciente (por ej. a su estadio del cambio) ${ }^{36}$. Sin embargo, la utilización óptima de la IB y su máxima rentabilidad (coste-beneficio) aún no han sido definidas.

Atribuyéndole un beneficio individual e incluso un posible efecto sobre la salud pública a través de repercutir sobre niveles agregados de beneficios, habría que procurar ampliar el espectro terapéutico engarzando distintas intervenciones entre sí, ajustando la profundidad y especificidad de la intervención a la gravedad del caso. No deberíamos confundir la ampliación de la base del tratamiento (IB para bebedores de riesgo) con el estrechamiento de su vértice (IB para los alcohol-dependientes) ${ }^{46}$. La creencia de que todas las intervenciones son eficaces podría llevarnos a engaño principalmente por favorecer ${ }^{5}$ la persistencia en la aplicación de intervenciones ineficaces, así como socavar la asignación eficaz de recursos por una exagerada generalización en detrimento de la provisión de programas específicos La evidencia acerca de la eficacia de las IB es muy superior por lo que respecta a las personas que no buscan tratamiento (intervenciones oportunistas) que para el ámbito especializado, 
donde habría que dar preferencia a tratamientos más sofisticados ${ }^{54}$. Como estrategia de intervención precoz, la IB breve sería rentable, no sólo por su eficacia intrínseca, sino también por el efecto multiplicativo de la misma que, reiterada desde el mismo o diversos ámbitos en sucesivas ocasiones sobre el mismo sujeto, evitaría el efecto de extinción del mensaje. La dicotomía entre un modelo individual y comunitario de prevención tiende a difuminarse. Las IB, junto con el tratamiento de enfermos, debería tener una repercusión sobre la salud pública tanto más manifiesto cuanto más integrado esté el programa en los recursos comunitarios. Entre los mayores incentivos para aplicar la IB, los médicos generalistas citan la disponibilidad de adecuados recursos de soporte y la eficacia probada de las intervenciones. Es importante darles a conocer su rendimiento y formarlos para su aplicación sin venderles panaceas que puedan hacerles sentir estafados. Al redimensionar cada intervención ganamos en credibilidad y aumenta la probabilidad de la adopción del modelo.

\section{BIBLIOGRAFÍA}

(1) * Miller WR, Brown J, Simpson TL, Handmaker NS, Bien TH, Luckie LF, Montgomery HA, Hester RK, Tonigan JS: What works? A methodological analysis of the alcohol treatment outcome literature. En Hester RK \& Miller WR (eds.): Handbook of Alcoholism Treatment Approaches: Effective Alternatives. Massachusetts: Allyn \& Bacon, 1995 (pp.: 12-44)

(2)** Bien, Th.; Miller, W.R.; Tonigan, J.S.: Brief interventions for alcohol problems: a review. Addiction 1993; 88: 315-336.

(3)* Wilk Al; Jensen NM; Havighurst TC: Metaanalysis of randomized control trials assessing brief interventions in heavy alcohol drinkers. $J$ Gen Int Med 1997; 12: 274-283.

(4)** Kahan M, Wilson L, Becker L: Effectiveness of physician-based interventions with problem drinkers: a review. Can Med Assoc J 1995; 152: 851-859.
(5)* Poikolainen K. Effectiveness of brief interventions to reduce alcohol intake in primary health care populations: A meta-analysis. Preventive Medicine 1999; 28: 503-509.

(6) Orford J, Edwards G: Alcoholism: A comparison of treatment and advice, with a study of the influence of marriage. Maudsley Monographs $n^{\circ}$ 26. Oxford: Oxford Univss, 1977.

(7) ** Heather N: Brief Intervention Strategies. En Hester RK \& Miller WR (eds.): Handbook of Alcoholism Treatment Approaches. Effective Alternatives. Boston, London Allyn \& Bacon, 1995 (pp.: $105-122$ ).

(8)** Wallace P, Cutler S, Haines A.: Randomised controlled trial of general practitioner intervention in patients with excessive alcohol consumption. Br Med J 1988; 297:663-668.

(9) Scott, E.; Anderson, P.: Randomized controlled trial of general practitioner intervention in women with excessive alcohol consumption. Drug and Alcohol Review 1991; 10: 313-321.

(10) Anderson, P.; Scott, E.: The effect of general practitioner's advice to heavy drinking men. $\mathrm{Br}$. J. Add 1992; 87: 891-900.

(11) Fleming MF, Barry KL,Manwill LB, et al: Brief physician advice for problem drinkers: A randomized controlled trail in community based primary care practices. JAMA 1997;25:52-71

(12) Kristensson H, Ohlin H, Hulten-Nosslin MB, Trell E, Hood B. Identification and intervention in heavy drinking in middle-aged men: results and follow-up of 24-60 month of long-term study with randomized controls. Alcoholism: Clin and Exp Res; 1983, 7: 203-209.

(13) Romesljö A, Andersson L, Barrner H, Borg S, Granstrand C, et al.: Randomized study of secondary prevention of early stage problem drinkers in primary health care. Br J Add 1989; 84: 1319-1327.

(14) Nilssen O:The Tromso study: identification of a controlled intervention on a population of earlystage risk drinkers. Prev Med 1991; 20: 518528.

(15) D'Onofrio G, Bernstein E, Bernstein J, Woolard R, Brewer Ph A, Craig SA et al.: Patients with alcohol problems in the emergency department, part 2: Intervention and referral. Academic Emerg Med 1998; 5: 1210-1217.

(16) DiClemente, CC. "Motivational interviewing and the stages of change." En Miller WR, Rollnick S (eds) Motivational interviewing. New 
York, London, The Guiford Press, 1991 (pp. 191202).

(17) Saunders JB, Lee NK: Hazardous alcohol use: its delineation as a subthreshold disorder, and approaches to its diagnosis and management. Comprehensive Psychiatry 2000, 41 (2), Suppl. 1: 95-103.

(18) Miller WR, Sánchez VC: Motivating young adults for treatment and lifestyle change. En: Howard G \& Nathan (eds.): Alcohol use and misuse by young adults. Notre Dame IN, University of Notre Dame Press 1993 (pp: 55-81).

(19) World Health Organization Brief Intervention Study Group. A cross national trial of brief interventions with heavy drinkers. Am J Public Health 1996; 86: 948-955.

(20.) IsraelY, Hollander O, Sánchez-Craig M, Booker $\mathrm{S}$, Miller $\mathrm{V}$ et al.: Screening for problem drinking and counseling by the primary care physician-nurse team. Alcoholism: Clinical \& Experimental Research 1996; 20: 1443-1450.

(21) Richmond R; Anderson P. Research in general practice for smokers and drinkers in Australia and the UK.I. Interpretation of the results. Addiction 1994; 89: 35-40.

(22) Babor TF, Grant M (eds.) Programme on Substance Abuse. Project on identification and management of alcohol-related problems. Report on Phase II: a randomized clinical trial of brief intervention in primary health care. Geneva, World Health Organization, 1992.

(23) Fleming MF, Manwell LB, Barry KL, Adams W, Stauffacher EA. Brief physician advice for alcohol problems in older adults: a randomized community-based trial. J Fam Pract 1999; 48 (5): 378-384.

(24) Gentinello, LM, Rivara FP, Donovan DM, Jurkovich GJ, Daranciang E et al.: Alcohol Interventions in a trauma center as a means of reducing the risk of injury recurrence. Ponencia al $119^{\text {th }}$ Meeting of the American Surgical Association, San Diego, 1999.

(25) Chick J, Ritson B, Connaughton J, Stewart A, Chick J: Advice versus extended treatment for alcoholism: a controlled study. Br J Add 1988; 83: 159-170.

(26) Antti-Poika I, Karaharju E, Roine R\& Salaspuro $\mathrm{M}$ : Intervention of heavy drinking: a prospective and controlled study of 438 consecutive injured male patients. Alcohol \&Alcoholism 1988; 23: 115-121.
(27) Richmond R, Heather N, Wodak A, Kehoe L, Webster I: Controlled evaluation of a general practice-based brief intervention for excessive drinking. Addiction 1995; 90: 119-132.

(28)* Altisent, R.; Córdoba, R.; Delgado, MT; Pico, MV.; et al.: Estudio multicéntrico sobre la eficacia del consejo para la prevención del alcoholismo en Atención Primaria EMPA. Med Clín, 1997; 109: 121-124.

(29)* Còrdoba R, Delgado MT, Pico V, Altisent R, Fores D, Monreal A, Frisas O, López del Val, A: Effectiveness of brief intervention on nondependent alcohol drinkers (EBIAL): a Spanish Multi-centre study. Family Practice 1998; 15 (6): 562-568.

(30) Heather, N.; Campion, P.D.; Neville, R.G.; Mac Cabe, D.: Evaluation of a controlled drinking minimal intervention for problem drinkers in general practice (The DRAMS scheme). J. Roy. Coll. Gen. Pract.1987; 37:358-363.

(31) Heather, N.: The public health and brief interventions for excessive alcohol consumption: The British experience. Addictive Behaviors 1996; 21 (6): 857-868.

(32) Colom J, Gual A. Un modelo de intervención integral sobre los problemas derivados del alcohol desde la atención primaria de salud: el programa Beveu Menys. JANO 2000 LIX: 9396.

(33) Fernández MI, Bermejo CJ, Alonso M, Herreros B, Nieto M, Novoa A, Marcelo MT: Efectividad del consejo médico breve para reducir el consumo de alcohol en bebedores. Atención Primaria 1997; 19 (3): 127-132.

(34) Diéz Manrique JF, García Usieto E. Programa Cantabria Intervención Breve. Santander: Gobierno de Cantabria. Dirección Regional de Bienestar Social y Dirección General de Salud Mental, 1999.

(35) Miller WR, Sovereign RG, Krege B.: Motivational interviewing with problem drinkers: I. The Drinker's Check-up as a preventive intervention. Behavioural Psychotherapy 1988; 16: 251268.

(36) Heather N, Rollnick S, Bell A, Richmond R: Effects of brief counseling among male heavy drinkers identified on general hospital wards. Drug Alcohol Rev 1996; 15: 29-38.

(37) Chick J: Early intervention for hazardous drinking in the general hospital. Alcohol Suppl, 1991;1: 477-479. 
(38) Chafetz ME, Blane HT, Abram HS, Golner J, Lacey $\mathrm{E}$ et al.: Establishing treatment relations with alcoholics. J Nerv Ment Dis 1962; 134 : 395-409.

(39) Bernstein E, Bernstein J, Levenson S: Project ASSERT: an ED based intervention to increase access to primary care, preventive services, and the substance abuse treatment system. Ann. Emerg Med 1997; 30: 181-189.

(40) Sommers MS, Dyehouse JM, Howe SR, Lemmink J.: Preventing alcohol-related motor vehicle injury with brief intervenction strategies: preliminary findings with 60 subjects. $42^{\text {nd }}$ Annual Proceedings. Association for the Advancement of Automotive Medicine. October 5-7, 1998. Charlottesville, Virginia.

(41) Sommers MS, Dyehouse JM, Howe SR, Weeks A, Russell AC: Using brief interventions following alcohol-related motor vehicle crashes to reduce drinking and further injury. Ponencia presentada en ICADTS-2000, Estocolmo, 2025 Mayo 2000.

(42) Longabaugh R, Minugh PA, Nirenberg TD, Clifford PR, Becker B, Woolard R. Injury as a motivator to reduce drinking. Acad Emerg Med 1995; 2: 817-825.

(43) Rodríguez-Martos A, Plasencia A, Escayola, M, Martí J, Ferrando J, Torralba LI. Intervención breve sobre accidentados con alcoholemia positiva desde un centro de traumatologia. (Adicciones 2001; 13 (4), en prensa).

(44) Drummond DC, Thom B, Brown C, Edwards G, Mullan MJ. Specialist versus general practitioner treatment of problem drinkers. The Lancet 1990; 336: 915-918.

(45) Richmond R, Kehoe L, Hailstone S, Wodak A, Uebel-Yan m: Quantitative and qualitative eva- luations of brief interventions to change excessive drinking, smoking and stress in the police force. Addiction 1999; 94 (10): 1509-1521.

$(46)^{*}$ Drummond DC: Alcohol interventions: do the best things come in small packages? Addiction 1997; 92: 375-379.

(47) Richmond R; Anderson P. Research in general practice for smokers and drinkers in Australia and the UK.II. Representativeness of the results. Addiction 1994; 89: 41-48.

(48) Edwards AG, Rollnick S. Outcome studies of brief alcohol intervention in general practice: the problem of lost subjects. Addiction 1997; 92: 1699-1704.

(49) Higgins-Biddle JC, Mullahy J, Daniels J, Mc Ree B. Alcohol screening and brief intervention: where research meets practice. Conn Med. 1997; 61 (9): 565-575.

(50) Holder H, Longabaugh R, Miller WR \& Rubonis $\mathrm{AV}$ : The cost-effectiveness of treatment for alcoholism: A first approximation. J Stud on Alc 1991; 52: 517-540.

(51)* Ballesteros J: ¿Es eficaz la intervención breve en bebedores a riesgo en el ámbito de la atención primaria? Una revisión sistemática de estudios españoles. Trastornos adictivos 2000; 2 (1): 32-40.

$(52)^{*}$ Nuffield Institute for Health: Brief interventions and alcohol use. Effective Health Care. Bulletin 1993;7.

(53) Delgado-Rodríguez M, Sillero-Arenas M. Sesgos en el metaanálisis. Med Clín (Barc) 1999; 112 (Supl 1): 43-50.

$(54) * *$ Heather N: Interpreting the evidence on brief interventions for excessive drinkers: the need for caution. Alcohol Alcohol 1995; 30: 287-296. 